\title{
Which foetal pelvic variables are useful for predicting right-rotational or left-rotational birth?
}

\author{
Pierre Frémondière ${ }^{1,2 *}$ \\ ${ }^{1}$ EU3M Aix-Marseille University, North department, bld Pierre-Dramard, 13916 Marseille, France \\ ${ }^{2}$ CNRS, EFS, ADES UMR 7268, Aix-Marseille University, faculty of medicine, North department, bld Pierre-Dramard, 13916 Marseille, France
}

\begin{abstract}
Objective: To assess the variables useful to predict right-rotational birth (ROA) and left-rotational birth (LOA), through the analysis of a large number of foetalpelvic variables, using discriminant analysis.

Materials and methods: One hundred and two pregnant women were included in this single centre prospective study. For each mother-foetus pair, 43 pelvic and 18 foetal variables were measured. Partial least squares-discriminant analysis was performed to identify foetal-pelvic variables that could statistically separate the 3 delivery modality groups: non-rotational birth or occiput anterior $(\mathrm{OA})$, right-rotational birth and left-rotational birth.

Results: For the OA versus LOA model, the most efficient variable for discrimination was the inlet-mid-plane angle. For the OA versus ROA model, the most important variable was the sacral overhang. For both model, we found that women with small foetuses, anthropoï inlet, reduce posterior space of the mid-plane and reduce oulet were more likely to have non-rotational birth.

Conclusion: This analysis helps us in identifying foetal-pelvic conditions to the rotation in physiological cases. It should be relevant to perform a similar work with cases of posterior position during explusion stage.
\end{abstract}

\section{Introduction}

During the birth process, the rotation of the foetal head is one of the most crucial steps of the birth mechanism for a successful delivery. Absence of complete rotation in case of LOP (left occiput posterior) or ROP (right occiput posterior) could lead to malposition and operative obstetric intervention for delivery [1]. At the beginning of the twenty century, an abondant literature about the mechanism of rotation is available [2-4]. But until several decades, only few studies explore the variables explaining position and rotation of the foetal head. For Sizer and Nirman [5], a computer literature search about occipitoposterior presentation for the period 1970 to 1999 yields very few references and only two studies examine possible associated factors. Moreover, these studies only consider obstetrical factors, i.e., incidence of premature rupture of membrane, induction of labour [6] or the length of labour [7] as possible associated factors rather than anatomical factors. Briggs [8] focuses on pelvis shape and finds that pelvic deformity might predispose to malposition. The aim of this study is to analyse the variability of the foetal-pelvic relationship from an anatomical perspective and its consequences on the foetal head position and to determine which pelvic and foetal variables could predict foetal head rotation.

\section{Materials and methods}

\section{Sample and foetal-pelvic variables}

One hundred and two women at Saint Joseph Hospital, Marseille, France, were recruited from 29 March 2011 to 10 December 2013 for this single-centre study. The women were recruited from 10,597 deliveries in the hospital. The inclusion criterion was birth at term with the foetus in a cephalic presentation. Exclusion criteria were cesarean delivery $(n=97)$, twin pregnancies $(n=15)$. The other deliveries excluded from the study were 10,198 with no CT scan, 133 for whom the CT scan was not available, 52 with breech presentation. The 102 deliveries included in the study were spontaneous vaginal delivery in 47 cases and instrument-assisted delivery in 55 cases. All 102 women had both epidural anaesthesia and a pelvic scan, i.e., radiological measurement of the parameters of the pelvis, before delivery. Foetopelvic data were from a previous study [9]. For each woman, we noted the foetal head orientation during the engagement and the explusion stage, maternal postures during the labour and expulsion, and the amount of ropivacaine during labour. The centre has 3 protocols for pelvic scanning, based on patient adiposity: low $(100 \mathrm{kV}, 25 \mathrm{~mA})$, standard $(100 \mathrm{kV}, 35 \mathrm{~mA})$, and high $(120 \mathrm{kV}, 35 \mathrm{~mA})$ adiposity. These 3 protocols produce low-level irradiation in the range of 15-35 $\mathrm{mGy} / \mathrm{cm}$. Indications for a pelvic scan were a scarred uterus, a breech presentation (but cephalic presentation at the beginning of labour), and suspicion or a history of foetal-pelvic disproportion. All patients enrolled in the study had the benefits/risks and long-term risks of CT scanning explained and all gave their consent for the scanning. Scans were performed with a 16-slice Siemens SOMATOM Definition Flash strip scanner located in the Medical Imaging Department of our hospital. The intersection gap was $0.6-1 \mathrm{~mm}$. All the pelvic diameter and angle were measured with Amira 5.0.0 software (FEI Visualization

Correspondence to: Pierre Fremondiere, UMR 7268 Faculty of Medicine, Secteur Nord Batiment A, CS80011 Bd Pierre Dramard 13344 Marseille Cedex 15. France, Tel: +33 4916988 90, Fax: +33 4916080 62, E-mail. pierrefremondiere@yahoo.fr

Key words: rotational birth, pelvimetry, orientation, birth mechanism, pelviscan, anthropometry

Received: July 24, 2017; Accepted: August 28, 2017; Published: September 01, 2017 
Sciences Group, Zuse Institute Berlin). A total of 43 pelvic variables were considered (Figure 1). Measurements with a standard error of $\leq$ $4 \mathrm{~mm}$ are deemed accurate and those outside this range considered inaccurate $[9,10]$. The newborn measurements were performed during the postpartum period using anthropometric tools (a cephalometric compass, a tape mesure, and a newborn scale). Eighteen foetal variables were measured (Figure 1). This study was approved by the South Mediterranean II Ethical Committee for the Protection of Persons and written informed consent was obtained from all the patients.

\section{Statistical analyses}

To identify relevant variables in the prediction of the rotational birth, partial least squares-discriminant analysis (PLS-DA) was used. This is commonly performed to statistically separate 2,3 or 4 groups by simultaneously using a large number of predictors common to both groups, i.e., in this case, the foetal-pelvic variables [11] The value of the Variable Importance in the Projection (VIP) quantified for the usefulness of each independent variable, allowing for discrimination between groups; only foetal-pelvic variables with a VIP value of $>1$ were considered useful for discrimination [12]. The statistical analyses were performed in two steps: a first PLS-DA identified variables with a VIP value of $>1$ and a second PLS-DA included only these variables. In this study, 7 models of combination comparing the different types of foetal head position were considered. Only PLS-DA with a $\mathrm{Q}^{2}$ value > $9,75.10^{-2}$ were presented [12]. The significance of differences between the groups was tested using the Mann-Whitney test. The $\mathrm{p}$ value $<0.05$ was considered statistically significant. Statistical tests were performed using XLSTAT 2013.1.02 software (Addinsoft, 2013).

\section{Results}

Table 1 shows maternal positions and amount of ropivacain during labour. The only significant difference was the right lateral decubitus position, more frequent in the instrument-assisted group than the spontaneous delivery group. Only one woman had a left
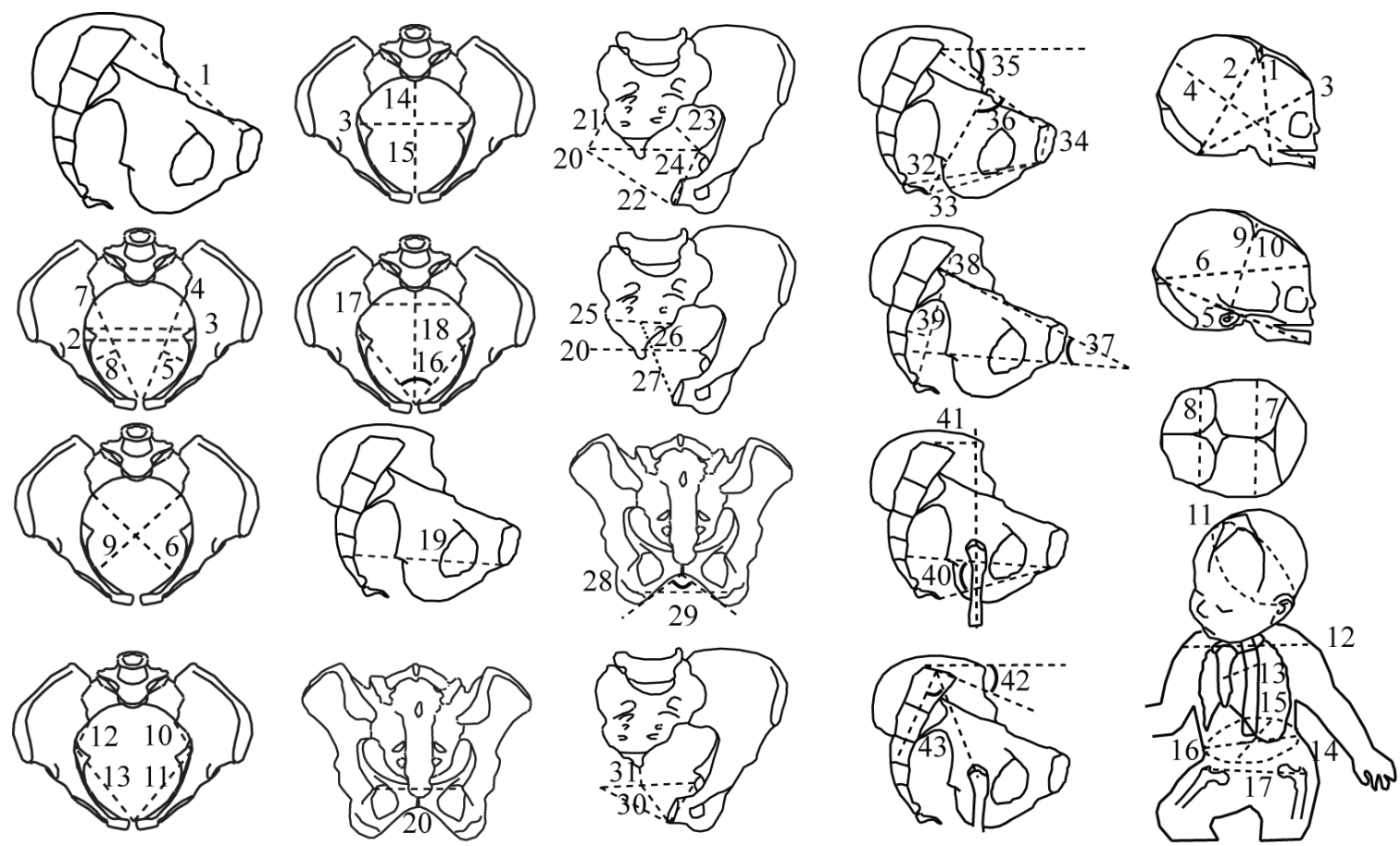

Figure 1. Foetal-pelvic variables

$\underline{\text { Pelvis; }}$

1: Obstetric conjugate (oc)

2: Medial transverse inlet (meti)

3: Maximal transverse inlet (mati)

4: Left ilio-pectineal chord length(licl)

5: Left ilio-pectineal chord subtense (lics)

6: Right oblique inlet (roi)

7: Right ilio-pectineal chord length (ricl)

8: Right ilio-pectineal chord subtense (rics)

9: Left oblique inlet (loi)

10: Left inlet posterior space (lips)

11: Left inlet anterior space (lias)

12: Right inlet posterior space (rips)

13: Right inlet anterior space (rias)

14: Sagittal posterior inlet (spi)

15: Sagittal anterior inlet (sai)

16: Pectineal angle (pa)

17: Inlet sacral breadth (isb)

18: Inlet antero-posterior (iap)

19: Mid-plane antero-posterior (map)

20: Interspinous (isp)

21: Right mid-plane posterior space (rmps)

22: Right mid-plane anterior space ( $\mathrm{rmas}$ )

23: Left mid-plane posterior space (lmps)

24: Left mid-plane anterior space (lmas)

25: Mid-plane sacral breadth (msb)

26: Sagittal posterior mid-plane (spm)

27: Sagittal anterior mid-plane (sam)

28: Transverse outlet (tout)

29: Subpubic angle (spa)

30: Sagittal anterior outlet (sao)

31: Sagittal posterior outlet (spo)

32: Outlet antero-posterior (oap)

33: Pubococcygeus lenght ( pcl)

34: Pubic symphysis height (psh)

35: Obstetric conjugate slope (ocs)

36: Obstetric conjugate-Umbiliccoccygeal angle (ocua)

37: Inlet-mid-plane angle (ima)

38: Sacral chord length ( $\mathrm{scl}$ )

39: Sacral chord subtense (scs)

40: Mid-plane-outlet angle (moa)

41: Sacral overhang (over)

42: Sacral slope (sslop)

43: Sacral incidence (inc)
Foetus;

1: submentobregmatic (smb)

2: suboccipitobregmatic (sob)

3: suboccipitofrontal (sof)

4: mentovertical (mv)

5: mento-occipital (moc)

6: occipitofrontal (of)

7: biparietal (bip)

8: bitemporal (bit)

9: right tragion-bregma ( $\mathrm{rtb})$

10: left tragion-bregma (ltb)

11: suboccipitobregmatic circumference (soc)

12: biacromial (bia)

13: sternum-thoracic vertebral (stv)

14: abdominal circumference (abdc)

15: abdominal sagittal (abds)

16: transverse abdominal (abdt)

17: bitrochanteric (bih)

18: birthweight (wght, not on figure) 
Table 1. Maternal postures and amount of ropivacaine during labour

\begin{tabular}{|l|l|l|l|l|}
\hline Variables & $\begin{array}{l}\text { Spontaneous } \\
\text { delivery }^{*} \\
\text { (n=44) }\end{array}$ & $\begin{array}{l}\text { Instrument- } \\
\text { assisted } \\
\text { delivery } \\
(\mathbf{n = 5 5 )}\end{array}$ & $\begin{array}{l}\text { Total } \\
\text { (n=99) }\end{array}$ & p value \\
\hline Ropivacaine (mg) & $39(21)$ & $37(17)$ & $38(19)$ & 0,69 \\
\hline supine position (\%) & $42(32)$ & $37(26)$ & $39(28)$ & 0,47 \\
\hline left lateral decubitus position (\%) & $21(24)$ & $25(20)$ & $23(21)$ & 0,15 \\
\hline Right lateral decubitus position (\%) & $7(15)$ & $12(15)$ & $10(14)$ & 0,02 \\
\hline squatting position (\%) & $20(24)$ & $14(16)$ & $17(20)$ & 0,38 \\
\hline Free to walk (\%) & $1(3)$ & $1(6)$ & $1(5)$ & 0,12 \\
\hline All fours position (\%) & $2(8)$ & $4(10)$ & $3(9)$ & 0,58 \\
\hline Hands-and-knees position (\%) & $7(7)$ & $6(5)$ & $6(6)$ & 0,93 \\
\hline
\end{tabular}

*for 3 patients, informations were not available. Values are expressed as mean (SD)

lateral decubitus position during expulsion. Table 2 shows the results of the 7 combinations in regard to the different foetal head position and with the related $\mathrm{Q}^{2}$ value. All women had OA (Occiput Anterior) position during the explusion stage. During the engagement, the most frequent foetal head position was the LOA (Left Occiput Anterior) $(\mathrm{n}=36)$, followed by the OA $(\mathrm{n}=28)$, ROA (Right Occiput Anterior) $(\mathrm{n}=18)$, ROP $(\mathrm{n}=12)$, Transverse position $(\mathrm{n}=6)$ and LOP $(\mathrm{n}=2)$. Two combinations had a $\mathrm{Q}^{2}>9,75.10^{-2}$ : the combination 1 (OA vs LOA) and the combination 2 (OA vs ROA). Figure 2 shows the classification of the foetal-pelvic variables in the combination 1 (OA vs LOA). Of the 24 important variables, $8(33 \%)$ were foetal variables, with right tragion-bregma being the most important variable. Large foetuses were more likely to belong to the rotational birth group (i.e., LOA) (see the variables smb, sof, mv, moc, rtb, bit, stv). Of the remaining $16(67 \%)$ variables, 5 (21\%) corresponded to pelvic features defined by the inlet variables, $5(21 \%)$ by the mid-plane, $3(12 \%)$ by the outlet variables and $3(12 \%)$ variables were related to the curve of the birth canal. The most important variable was the inlet-midplane angle. Women with anthropoïd (i.e., oval pelvis with the anteroposterior diameter greater than transverse diameter) inlet (oc, spi, iap, ricl, rias), a large anterior space of the mid-plane (sam, rmas, lmas), but a reduce posterior space of the mid-plane (rmps, lmps), a straight birth canal (ima, ocua) but a curved sacrum (scs), a reduce outlet ( $\mathrm{pcl}$ ) especially the posterior space (sao) were more likely to had non-rotational birth (i.e., OA).

Figure 3 shows the classification of the foetal-pelvic variables in the combination 2 (OA vs ROA). Of the 18 important variables, 1 (5\%) was a foetal variable: the bitrochanterian diameter. Foetuses with large bitrochanterian diameter were more likely to belong to rotational birth group (i.e., ROA). Of the remaining 17 variables, $5(28 \%)$ corresponded to pelvic features defined by the mid-plane, $4(22 \%)$ by the inlet, 4 (22\%) were related to the curve of the birth canal, 2 (11\%) were outlet variables, and $2(11 \%)$ were related to the pelvic balance in standing position. The most important variable was the sacral overhang. Women with anthropoïd inlet (meti, mati, lics, rics), a reduce mid-plane (map, isp), especially the posterior space (spm, rmps, lmps), a straight birth canal (ima, ocs, scl, psh), a reduce outlet (tout, spa) were more likely to had non-rotational birth (i.e., OA).

\section{Discussion}

In this study, we found that a small foetus and anthropoïd pelvic inlet were associated with non-rotational birth. These findings confirmed those of previous studies [13,14], in which it was reported that large foetuses were more constrained by the pelvic shape and had to rotate to fit with the birth canal [13]. For Caldwell and Moloy [14], anthropoïd pelvises were more likely to had OA orientation at inlet. These results support our findings.
Our results suggest that women with a reduce mid-plane, especially the posterior space were more likely to had non-rotational birth. This confirms the findings of Tague [15], who notes that a wide anterior space of the mid-plane is a requisite for the foetal head descent in case of OA position. The importance of a large posterior space of the mid-plane with regard to the mode of delivery had been pointed out in previous studies $[15,16]$. Selective pressure for an obstetrically wide pelvis is supposed to explain sexual dimorphism in modern humans. Recently, Brown [16] demonstrated the effect of sex and body mass

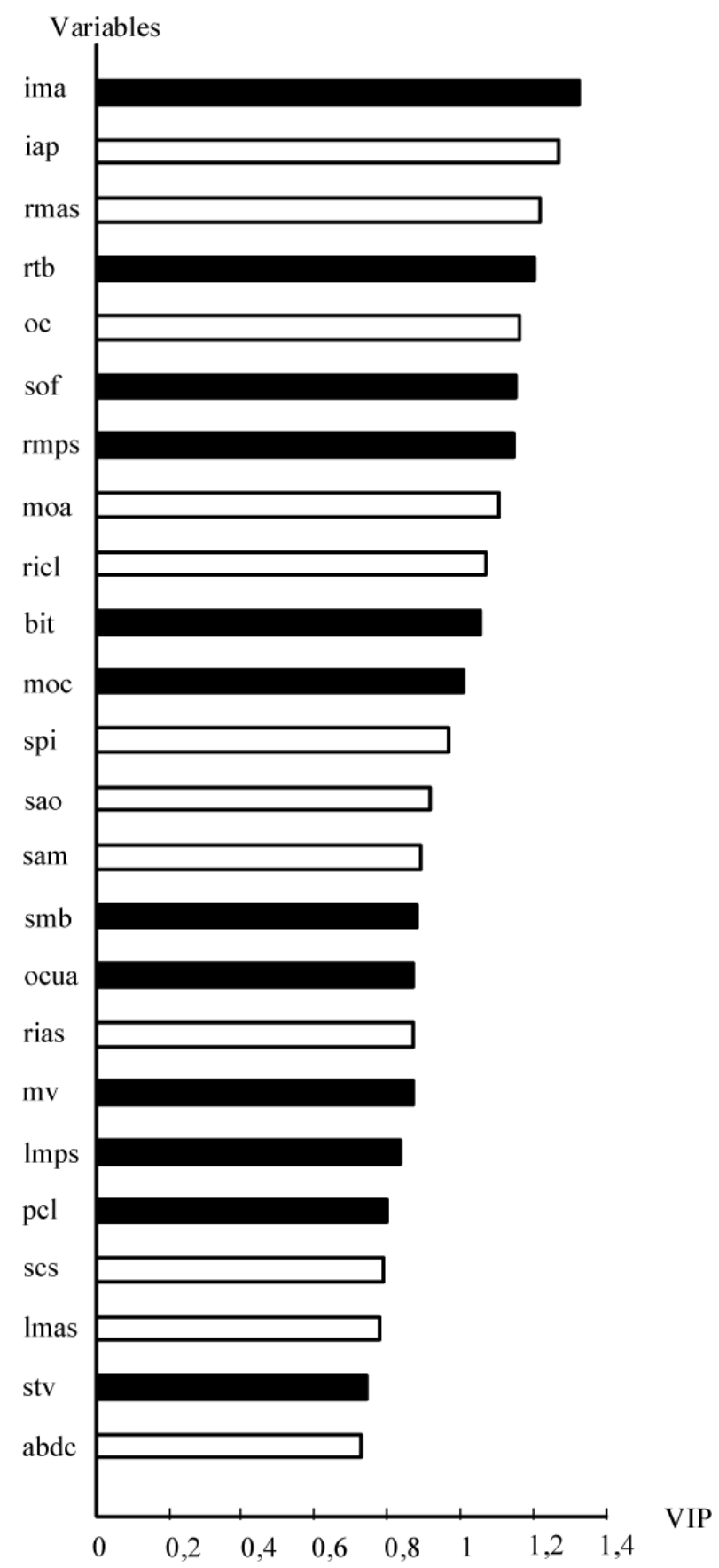

Figure 2. Classification of the variables in the OA versus LOA combination: 24 variables are important (i.e., with a variable importance in the projection $>1$ in the first PLS-DA). Black shows variables allocating mother-foetus pairs to the LOA group when their values were high. White shows variables allocating mother-foetus pairs to the OA group when their values were high. For explanation of abbreviations, see Figure 1. 
Table 2. Well-classified mother-foetus pairs according to partial least squares-discriminant analysis

\begin{tabular}{|c|c|c|c|}
\hline Combination & n & $\begin{array}{l}\text { Well-classification } \\
(\%)\end{array}$ & $\mathbf{Q}^{2}$ \\
\hline Combination $1^{*}$ & 64 & $69 \%$ & \\
\hline $\mathrm{OA}$ & 28 & $64 \%$ & 0.145 \\
\hline LOA & 36 & $83 \%$ & \\
\hline Combination $2^{*}$ & 46 & $81 \%$ & \\
\hline $\mathrm{OA}$ & 28 & $89 \%$ & 0.117 \\
\hline ROA & 18 & $72 \%$ & \\
\hline Combination 3 & 82 & $59 \%$ & \\
\hline OA & 28 & $57 \%$ & 0.047 \\
\hline LOA & 36 & $89 \%$ & \\
\hline ROA & 18 & $0 \%$ & \\
\hline Combination 4 & 42 & $71 \%$ & \\
\hline $\mathrm{OA}$ & 28 & $93 \%$ & -0.056 \\
\hline ROP & 12 & $33 \%$ & \\
\hline LOP & 2 & $0 \%$ & \\
\hline Combination 5 & 82 & $77 \%$ & \\
\hline $0^{\circ}$ & 28 & $46 \%$ & 0.077 \\
\hline $45^{\circ}$ & 54 & $91 \%$ & \\
\hline Combination 6 & 96 & $61 \%$ & \\
\hline $0^{\circ}$ & 28 & $36 \%$ & 0.019 \\
\hline $45^{\circ}$ & 54 & $89 \%$ & \\
\hline $135^{\circ}$ & 14 & $0 \%$ & \\
\hline Combination 7 & 102 & $54 \%$ & \\
\hline $0^{\circ}$ & 28 & $32 \%$ & 0.035 \\
\hline $45^{\circ}$ & 54 & $85 \%$ & \\
\hline $90^{\circ}$ & 6 & $0 \%$ & \\
\hline $135^{\circ}$ & 14 & $0 \%$ & \\
\hline
\end{tabular}

"PLSDA with $\mathrm{Q}^{2}>9.75 .10^{-2}$, OA: Occiput Anterior, LOA: Left Occiput Anterior, ROA Right Occiput Anterior, LOP: Left Occiput Posterior, ROP: Right Occiput Posterior, $0^{\circ}: \mathrm{OA}, 45^{\circ}: \mathrm{LOA}+\mathrm{ROA}, 90^{\circ}=$ Left occiput transverse + right occiput transverse, $135^{\circ}$ : LOP+ROP.

on distances in both the anterior and the posterior spaces of the pelvis. Her results suggest that the shape and size of the posterior space is predominantly influenced by sex, and thus by obstetrics.

We found that women with a reduce outlet were more likely to had non-rotational birth. A plausible explanation is the presence in our sample of women with "funnel-shaped" pelvis. For Cadwell and Moloy [14], a "funnel-shaped" pelvis has a large obstetric conjugate because of the backward position of the $1^{\text {st }}$ sacral vertebra, but a reduce outlet because of the forward position of the $5^{\text {th }}$ sacral vertebra. The OA position during the engagement is more appropriate in the "funnel-shaped" pelvic because of the large obstetric conjugate and the anthropoïd shape. Indeed, this pelvis should be associate with nonrotational birth (persistence of OA position during birth) and with a reduce outlet.

Our results suggested that women with a curved birth canal were more likely to had rotational birth. Most of authors suggest that the rotation of the foetal head is related to the descent movement [17]. We suggest that a curved birth canal increase the timing for the descent step, and should therefore support the rotation.

Many factors could contribute to change birth canal configuration during labour. Among them, birth position is supposed to have a crucial impact. It is impossible to perform a CT-scan during each stage of the labour given the high risk of radiation exposition of the foetus due to the procedure. In our sample, only the right lateral decubitus position differs significantly between the instrument-assisted group and the spontaneous delivery group. This position is not supposed to change significantly the female pelvic bony, contrary to the handsand-knees and squatting positions [18]. We could therefore suggest a minimal impact of birth position in the delivery outcomes.

In our sample, the instrument-assisted birth rate is high. This high rate should be explained by the systematic use of an epidural anesthesia. In this study, it is impossible to compare the delivery outcomes between epidural vs no-pharmacic method pain relief because all women had an epidural anesthesia. However, we compare the amount of ropivacain between spontaneous and assisted delivery. Our results suggest no significant difference between these two groups. We show in a previous study [9] that fetal-pelvic relationship could explain delivery outcomes, and among them, instrumental assistance: big fetuses and women with a small antero-posterior outlet diameter, a large obstetric conjugate, and a narrow pubic arch were at a greater risk of requiring instrumental assistance.

\section{Variables}

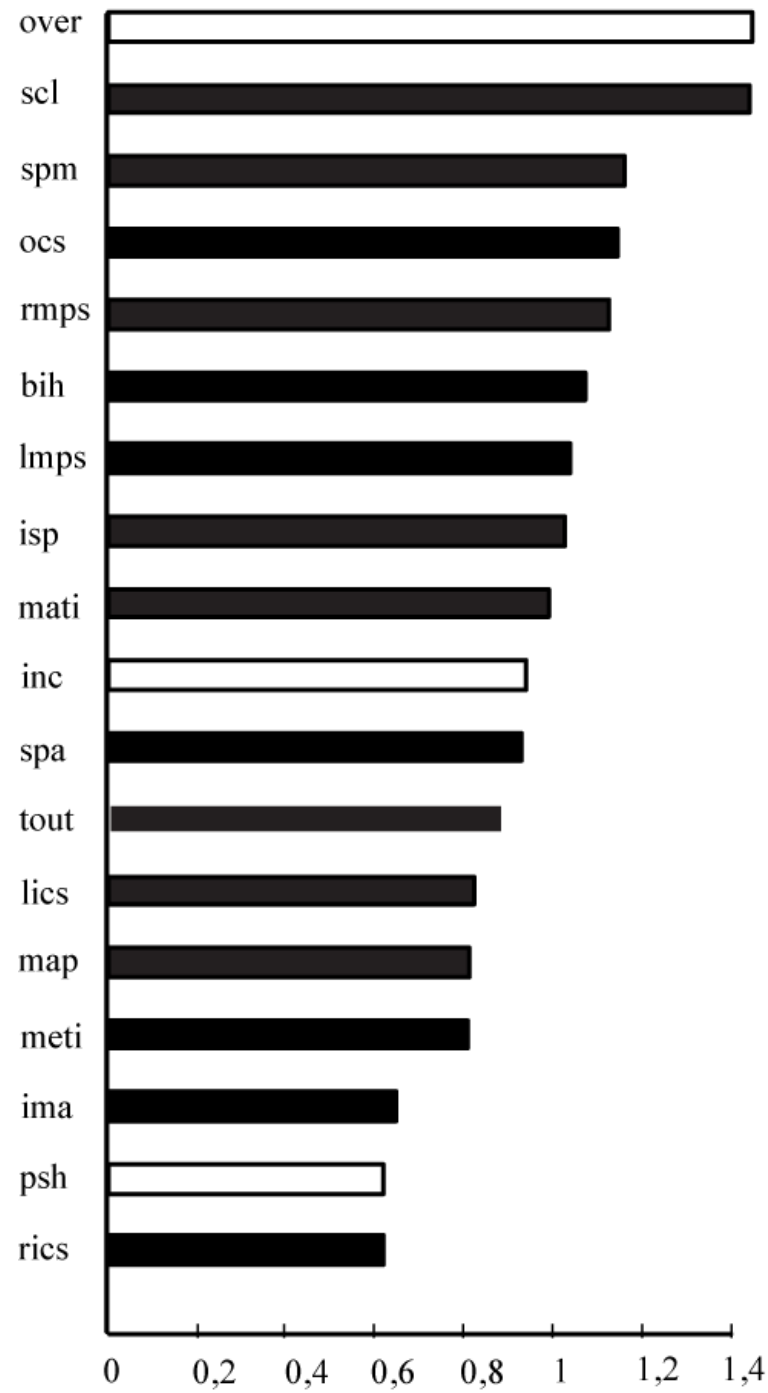

Figure 3. Classification of the variables in the OA versus ROA combination: 18 variables are important (i.e., with a variable importance in the projection $>1$ in the first PLS-DA) Black shows variables allocating mother-foetus pairs to the ROA group when their values were high. White shows variables allocating mother-foetus pairs to the OA group when their values were high. For explanation of abbreviations, see Figure 1. 


\section{Conclusion}

We found that women with small foetuses, anthropoïd inlet, reduce posterior space of the mid-plane and reduce oulet were more likely to have non-rotational birth. In clinical practice, digital examination of the pelvis should be combined with the determination of the foetal head position. We studied for the first time the foetal-pelvic relationship and its consequences on foetal head movement during the delivery. These findings improve our knowledge of the mechanism of the rotation during birth process. This study is observational and therefore remains a preliminary analysis of the mechanism of birth rotation. Further studies should consider more cases of mother-foetus pairs, or a control process of plausible confounding factors. Unfortunately, there is a lack of OP position cases at explusion stage in our sample. This position is related to birth injuries and maternal complications. Further analyses should consider cases of OP positions. This would improve our understanding of posterior rotation.

\section{References}

1. Gardberg M, Laakkonen E, Salevaara M (1998) Intrapartum sonography and persistent occiput posterior position: a study of 408 deliveries. Obstet Gynecol 91: 746-749. [Crossref]

2. Paramore RH (1909) A critical inquiry into the causes of the internal rotation of the foetal head. Brit J Obstet Gynaecol 16: 213-232.

3. Trillat P (1904) Du mecanisme des rotations intra-pelviennes de la tete. Theorie du plan lombo-iliaque. Ph.D. Faculte de medecine et de pharmacie de Lyon.

4. Varnier H (1888) From the lower muscular strait of the obstetrical pelvis, Paris, Steinheil G.

5. Sizer AR, Nirmal DM (2000) Occipitoposterior position: associated factors and obstetric outcome in nulliparas. Obstet Gynecol 96: 749-752. [Crossref]
6. Neri A, Kaplan B, Rabinerson D, Sulkes J, Ovadia J (1995) The management of persistent occipito-posterior position. Clin Exp Obstet Gynecol 22: 126-131. [Crossref]

7. Gardberg M, Tuppurainen M (1994) Persistent occiput posterior position: A clinical problem. Acta Obstet Gynecol Scand 73: 45-47. [Crossref]

8. Briggs ND (1989) Outcome of labour in occipitoposterior positions in an African population. Br J Obstet Gynaecol 96: 1234-1236. [Crossref]

9. Fremondiere P, Thollon L, Adalian P, Delotte J, Marchal F (2017) Which Foetal-Pelvic Variables Are Useful for Predicting Caesarean Section and Instrumental Assistance? Med Princ Pract 26: 359-367. [Crossref]

10. Anderson N, Humphries N, Wells JE (2005) Measurement error in computed tomography pelvimetry. Australas Radiol 49: 104-107. [Crossref]

11. Perez-Enciso M, Tenenhaus M (2003) Prediction of clinical outcome with microarray data: a partial least squares discriminant analysis (PLS-DA) approach. Hum Genet 112: 581-592. [Crossref]

12. Tenehaus M (1998) PLS regression theory and practice, Paris, Technip editor.

13. Joulin M (1864) Comparative Anatomy and Physiology of the Mammalian Basin. Arch gen med 6: 29-46.

14. Caldwell WE, Moloy HC (1938) Anatomical Variation in the Female Pelvis: Their Classification and Obstetrical Significance. Proc R Soc Med 32: 1-30. [Crossref]

15. Tague RG (2000) Do big females have big pelves? Am J Phys Anthropol 112: 377-393 [Crossref]

16. Brown KM (2015) Selective pressures in the human bony pelvis: Decoupling sexual dimorphism in the anterior and posterior spaces. Am J Phys Anthropol 157: 428-440. [Crossref]

17. Pal A, Modak R (2013) Practical manual of obstetrics. India, Jaypee Brothers Medical Publishers.

18. Michel SC, Rake A, Treiber K, Seifert B, Chaoui R, et al. (2002) MR obstetric pelvimetry: effect of birthing position on pelvic bony dimensions. AJR Am J Roentgenol 179: 1063-1067. [Crossref]

Copyright: (C2017 Fremondiere P. This is an open-access article distributed under the terms of the Creative Commons Attribution License, which permits unrestricted use, distribution, and reproduction in any medium, provided the original author and source are credited. 